\title{
How multi-infarct encephalopathy reflects on cognitive functioning after first ever ischemic stroke?
}

\begin{abstract}
Introduction: Vascular dementia is heterogeneous entity with many important subtypes. The most frequent of them are post-stroke cognitive impairment and multi-infarct encephalopathy (MIE). The aim of our study was to examine the impact of MIE on poststroke cognitive impairment.
\end{abstract}

Material and methods: We examined 109 post-stroke survivors ( 30 without and 79 with additional MIE) on two stage model - at the $3^{\text {rd }}$ and at the $90^{\text {th }}$ day after the incident with Mini Mental State Examination, tests for nonverbal and verbal memory and for executive function.

Results: The performances on all tests were lower in MIE-group than those without MIE, although there were different models for cognitive improvement between the groups.

Conclusion: Stroke and MIE as types of vascular cognitive impairment have their own specific relations, associated with cognitive abilities. MIE is associated with poor cognitive performance for all important cognitive domains - memory and executive functions after stroke. Such patients are more risky for severe cognitive deterioration and poor outcome and require specific cognitive rehabilitation. New studies are important for highlighting the relationships between stroke and other types of vascular dementia in order to seek new treatment strategies orientated towards such cases.

Keywords: cognitive impairment, stroke, vascular dementia, multi-infarct encephalopathy
Volume 5 Issue 2 - 2018

\author{
Mirena Valkova \\ University hospital Sofiamed, Bulgaria
}

Correspondence: Mirena Valkova, University hospital “Sofiamed”, Sofia, Bulgaria, Email dr.plamenova@gmail.com

Received: March 06, 2018| Published: April 03, 2018

\section{Introduction}

The term "vascular cognitive impairment" was defined as cognitive dysfunction due to or associated with cerebro-vascular disease or vascular risk factors. ${ }^{1-4}$ The conception of vascular cognitive impairment originated on the basis of the idea of the possible reversibility of vascular dementia in its preclinical and early clinical stages and searching for appropriate therapies for its primary and secondary prevention and treatment especially in high-risk groups..$^{2,4,5}$ The role for such reversibility had processes as cognitive reserve and neuroplasticity. ${ }^{6-9}$ The most common variants of vascular cognitive impairment were postroke cognitive impairment and multi-infarct cognitive impairment. ${ }^{3,4}$

Post-stroke cognitive impairment is defined as any type of cognitive impairment developed 3 months after stroke. ${ }^{10-12}$ The major determinants of cognitive dysfunctions in subacute stroke stage are considered age, education, the severity, number and location of stroke, the severity of acute stroke cognitive impairment and coexistence of depression and other forms of vascular dementia., ${ }^{7,10,13,14}$ The impact of stroke on cognitive functioning is variable -some patients can show obvious decline, others can remain stable, or revert to baseline cognitive functioning. ${ }^{12}$

Multi-infarct encephalopathy (MIE) is the second leading cause for dementia after Alzheimer's disease. ${ }^{15}$ The historical term "multiinfarct dementia" was developed before the term of "vascular dementia" for all cognitive decline due to multiple lesions and infarcts in both white and gray matter that follow occlusions in cerebral arteries and arterioles. ${ }^{15,16}$ It is than incorporated as the most common group of vascular dementias. However the most prominent cognitive feature in this kind of dementia is considered executive dysfunction, although other cognitive domains are also at risk. ${ }^{15,17}$ Post-stroke cognitive impairment and MIE often co-occurred. The aim of our study was to examine the impact of MIE on post-stroke cognitive impairment.

Material and methods: We examined 109 patients (on average age $66.67 \pm 9.03$ years; 67 males and 42 females, 33 with basic, 62 with middle and 14 with high education, 30 without and 79 with additional MIE) with first ever ischemic stroke (with average severity on National Institutes of Health Stroke Scale (NIHSS) 9.23 \pm 2.50 p.), untreated with thrombolysis (all of them were coming at the second or third day after the first stroke symptoms). The inclusion criteria were existence of clinical and imaging verified acute ischemic stroke, NIHSS severity below 15 points, stroke size below $1 / 3$ of the hemisphere, ability to complete the using neuropsychological battery, consent for participating in the study, lack of historical, imaging and clinical data for other cerebral diseases, patients with MIE should be without historical data for previous acute stroke and without MIE lesions above $4 \mathrm{~mm}$, lack of other decompensate somatic diseases, lack of family history or history for dementia or psychiatric illnesses, lack of evidence for psychiatric signs during the examination, lack of aphasia, apraxia or agnosia during the examination, lack for anamnestic data for previous treatment with drugs that seriously involved cognitive functioning. Methods: We used historical data (clinical interview, previous medical documents, etc.), full neurological and somatic examination, laboratory data, electrocardiography and neuroimaging for diagnostic purposes. All appropriate patients were selected on the basis of study criteria and gave informed consent.

All selected individuals were examined at the third day after the incident via "acute" neuropsychological battery which consisted of 
Mini Mental State Examination (MMSE), 10 words Luria test and Isaacs Set Test for verbal fluency (VF). They all were examined again at the $90^{\text {th }}$ day after the incident with "wide" battery which consisted of MMSE, 10 words Luria Test, Isaacs Set Test, Blessed Dementia Information Memory Concentration test (subscales Information, Nonpersonal and Personal Memory), Benton Visual Retention Test (BVRT) and Wisconsin Card Sorting Test (WCST). 4 of patients with MIE refused to fulfill BVRT and one of them - WCST at the second stage of the study.

The analysis was done via description and correlation analysis, using ANOVA, MANOVA, Mann-Whitney u-test, t-test, paired t-test, Pearson $\mathrm{x}^{2}$. We used "STATGRAPHICS Plus 5.0", EDSS 19 and Microsoft Excel. All results were interpreted at $95 \%$ confidential level $(\mathrm{p}<0.05)$.

\section{Results}

a. Demographic data: Demographic characteristics of both groups are given at Table 1.

b. General cognitive performance: The overall MMSE results are given at Table 2. No significant differences were found between $3^{\text {rd }}$ and $90^{\text {th }}$ day MMSE performances in both groups.

Table I Demographic data

\begin{tabular}{lll}
\hline & Without MIE & With MIE \\
\hline & $\mathrm{n}=30$ & $\mathrm{n}=79$ \\
Average age (years) & $68.93 \pm 8.58$ & $65.52 \pm 8.98$ \\
Male:Female & $21: 9$ & $47: 32$ \\
NIHSS points & $2-15$ (Med. 8.0) & $\mathrm{I}-15$ (Med.7.0) \\
\hline
\end{tabular}

Table 2 MMSE results

\begin{tabular}{llll}
\hline & Without MIE & With MIE & \\
\hline & $\mathrm{n}=30$ & $\mathrm{n}=79$ & $\mathrm{P}=$ \\
MMSE at third day (p.) & $23.45 \pm 0.57$ & $19.28 \pm 0.52$ & $0.000 \mathrm{I}$ \\
MMSE at $90^{\text {th }}$ day (p.) & $24.44 \pm 0.53$ & $19.07 \pm 0.48$ & $0.000 \mathrm{I}$ \\
\hline
\end{tabular}

\section{Memory}

a. Verbal memory: Patients with additional MIE showed lower test results on 10 words test at $3^{\text {rd }}$ and $90^{\text {th }}$ day after the incident, although the mild improvement during the first three months on short-term memory. The group without MIE showed improvement on delayed recall, but not on working memory (Table 3). All results on long-term memory were poor in the group with MIE, however the most statistical significant difference was found for subscale "Information" (Table 4).

b. Visual memory: Patients without MIE had better results on visual memory, measured by BVRT on $90^{\text {th }}$ day after the stroke (Table 5)

\section{Executive functions:}

a. Verbal fluency: Patients with MIE showed poor verbal fluency both at $3^{\text {rd }}$ and $90^{\text {th }}$ day after the incident (Table 6); however they had better 3 month improvement (with average $2.77 \pm 0.32$ p.) vs patients without MIE (with average $1.43 \pm 0.35$ p.). b. Wisconsin card sorting test performance: Patients in MIE group had more errors and perseverations than those without (Table 7).

Table 3 Results on verbal memory

\begin{tabular}{llll}
\hline & Without MIE & With MIE & \\
\hline & $\mathrm{n}=30$ & $\mathrm{n}=79$ & $\begin{array}{l}\text { P(Without } \\
\text { MIE;With MIE) } \\
\text { STM I }\end{array}$ \\
STM 2 & $6.08 \pm 0.19$ & $4.92 \pm 0.19$ & $\mathrm{p}=0.000 \mathrm{I}$ \\
(STMI;STM2) & $\mathrm{p}>0.05$ & $5.00 \pm 0.16$ & $\mathrm{p}=0.000 \mathrm{I}$ \\
DR I & $4.58 \pm 0.28$ & $\mathrm{p}=0.0042$ & \\
DR 2 & $5.26 \pm 0.24$ & $3.35 \pm 0.26$ & $\mathrm{p}=0.0043$ \\
P(DR I;DR2) & $\mathrm{p}=0.0032$ & $\mathrm{p}>0.05$ & $\mathrm{p}=0.000 \mathrm{I}$
\end{tabular}

STM, short-term memory (working memory); DR, delayed recall; I-measured at $3^{\text {rd }}$ day after the incident, 2 - measured at $90^{\text {th }}$ day after the incident. All are measured by average words

Table 4 Results on memory subscales on BDIMCT at $90^{\text {th }}$ day after the incident

\begin{tabular}{llll}
\hline & Without MIE & With MIE & \\
\hline Subscales & $\mathrm{n}=30$ & $\mathrm{n}=79$ & $\mathrm{p}=$ \\
Information (p.) & $\mid 3.04 \pm 1.35$ & $10.23 \pm 1.8 \mathrm{I}$ & $0.000 \mathrm{I}$ \\
Nonpersonal memory (p.) & $2.20 \pm 0.8$ & $1.60 \pm 0.04$ & 0.0003 \\
Personal memory (p.) & $6.80 \pm 0.76$ & $6.25 \pm \mathrm{I} .13$ & 0.0040
\end{tabular}

Table 5 BVRT performances differences between patients with and without MIE at $90^{\text {th }}$ day after the incident

\begin{tabular}{|c|c|c|c|}
\hline & Without MIE & With MIE & \\
\hline & $n=30$ & $\mathrm{n}=75$ & \\
\hline Number of correct items & $2.73 \pm 1.75$ & $1.16 \pm 0.22$ & $\mathrm{p}=0.000 \mathrm{I}$ \\
\hline Number of Errors & $11.73 \pm 4.78$ & $16.68 \pm 4.09$ & $p=0.0001$ \\
\hline $\begin{array}{l}\text { Number of Errors at left } \\
\text { visual field }\end{array}$ & $6.37 \pm 3.78$ & $8.55 \pm 3.82$ & $p=0.0041$ \\
\hline $\begin{array}{l}\text { Number of Errors at right } \\
\text { visual field }\end{array}$ & $4.80 \pm 2.56$ & $6.91 \pm 2.00$ & $p=0.0001$ \\
\hline Number of Distortions & $3.29 \pm 1.74$ & $4.84 \pm 2.08$ & $\mathrm{p}=0.000 \mathrm{I}$ \\
\hline
\end{tabular}

The test was completed by 75 patients with MIE (4 patients refused to fulfil BVRT). The results from other BVRT subscales are not given, because of statistical problems

Table 6 Verbal fluency (measured by IST) performances

\begin{tabular}{llll}
\hline & Without MIE & With MIE \\
\hline & $n=30$ & $n=79$ & $\begin{array}{l}\text { P(withoutMIE; with } \\
\text { MIE) }\end{array}$ \\
VF I (p.) & $22.88 \pm 7.20$ & $13.55 \pm 7.00$ & $0.000 \mathrm{I}$ \\
VF 2 (p.) & $24.80 \pm 6.67$ & $\mathrm{I} 6.37 \pm 6.06$ & $0.000 \mathrm{I}$ \\
P(VFI;VF2) & $\mathrm{p}=0.0450$ & $\mathrm{p}=0.0003$ & \\
\hline
\end{tabular}

VF, verbal fluency; I-at $3^{\text {rd }}$ day; 2 - at $90^{\text {th }}$ day after the incident 
Table 7 Wiskonsin card sorting test performances of both groups at $90^{\text {th }}$ day after the stroke

\begin{tabular}{|c|c|c|c|}
\hline & $\begin{array}{l}\text { Without } \\
\text { MIE }\end{array}$ & With MIE & \\
\hline & $n=30$ & $\mathrm{n}=78$ & $p=$ \\
\hline Number of errors & $45.54 \pm|4.6|$ & $66.21 \pm 16.09$ & 0.0001 \\
\hline Number of perseverations & $30.92 \pm 16.98$ & $44.55 \pm 18.63$ & 0.0001 \\
\hline $\begin{array}{l}\text { Number of perseverative } \\
\text { errors }\end{array}$ & $23.28 \pm 11.48$ & $34.4 I \pm 14.93$ & 0.0001 \\
\hline $\begin{array}{l}\text { Number of nonperseverative } \\
\text { errors }\end{array}$ & $21.24 \pm 7.92$ & $31.07 \pm 13.09$ & 0.0001 \\
\hline $\begin{array}{l}\text { Number of trials to complete } \\
\text { the } \text { I }^{\text {st }} \text { category. }\end{array}$ & $28.78 \pm 13.04$ & $46.18 \pm 18.73$ & 0.0107 \\
\hline
\end{tabular}

I patient with MIE did not complete the test at $90^{\text {th }}$ day after the incident.

The groups had no statistically significant differences on table missing scales.

\section{Discussion}

Post-stroke dementia and MIE are in complex relationship with one another, although both of them are the most frequent subtypes of vascular dementia and share similar risk factors., ${ }^{3,5}$ However, patients with vascular risk factors seem to have lower cognitive reserve than those without, although the great heterogeneity of the first group ${ }^{18}$ More over as we have pointed before, patients with ischemic stroke seem to show significant intergroup differences with regard to the course of their post-stroke cognitive decline. ${ }^{12}$ Such intergroup heterogeneity is probably multifactorial conditioned and complex but one of the most important of them is possibly the additional association of MIE. MIE is associated with lower cognitive performance in all examined cognitive domains at acute and subacute stroke stage (verbal and nonverbal memory, executive functions and general cognitive performance), besides the similar demographics of our examined groups. Additional MIE is reflected also on the course of cognitive decline during the first three months after the stroke incident in some cognitive domains (working memory and verbal fluency).

\section{General cognitive performance}

Generally stroke survivors show lower MMSE performance than is expected in general population, ${ }^{19}$ having some kind of cognitive impairment, but those with MIE have lower results then those without independently from age or level of education. Moreover most of stroke survivors without MIE have MMSE scoring responding to minor neurocognitive disorder (NCD), but those with MIE have borderline scoring between minor and major NCD and these differences are kept for at least at 3 months after the incident.

Memory: Our result analysis supports the hypothesis that memory (especially working memory) is one of the most impaired cognitive domains after stroke, ${ }^{7,13,20}$ but patients with MIE again show lower performance than stroke survivors without. However, our groups have different models of improvement during the first three months after the incident. So during the first three months after the stroke patients without MIE show improvement in their episodic, but not working memory and vice versa for patients with MIE. However both shortterm memory and delayed recall results remain significantly worse in MIE positive group at acute and subacute stroke stage.

Long term memory (episodic and semantic) is poor in patients with MIE, but surprisingly the most significant intergroup difference is found not only for orientation (information) but for semantic (nonpersonal) memory.

Visual memory is also poor in MIE positive group which can be associated with additional impairment of visual-spatial abilities in this group.

Executive functions. Executive dysfunction is one of the key features of vascular dementia ${ }^{2,5,10,17}$ and both with stroke severity contribute most to poor functional outcome..$^{21}$ Patients with MIE have greater deficits in all basic executive functions (inhibitory control, working memory, attention and cognitive flexibility) than those without. ${ }^{22}$ However both of our groups have shown some improvement in verbal fluency (IST performance) during the first three months, although it is more statistically significant in MIE group, but even so, they show significantly lower performances than those of the MIE negative group.

\section{Conclusion}

Stroke and MIE as types of vascular cognitive impairment have their own specific relations, associated with cognitive abilities. MIE is associated with poor cognitive performance for all important cognitive domains - memory and executive functions after stroke. Such patients are more risky for severe cognitive deterioration and poor outcome and require specific cognitive rehabilitation. New studies are important for highlighting the relationships between stroke and other types of vascular dementia in order to seek new treatment strategies orientated towards such cases.

\section{Acknowledgement}

None.

\section{Conflict of interest}

The author declares that there is no conflict of interest.

\section{References}

1. Hachinski V, Iadecola C, Petersen RC, et al. National Institute of Neurological Disorders and Stroke-Canadian Stroke Network vascular cognitive impairment harmonization standards. Stroke. 2006;37(9):2220 2241 .

2. Stephan BC, Matthews FE, Khaw KT, et al. Beyond mild cognitive impairment: vascular cognitive impairment, no dementia (VCIND). Alzheimers Res Ther. 2009;1(1):4.

3. Engelhardt E, Tocquer C, André C, et al. Vascular dementia. Diagnostic criteria and supplementary exams. Recommendations of the Scientific Department of Cognitive Neurology and Aging of the Brazilian Academy of Neurology. Part I. Dement Neuropsychol. 2011; 5(4):251-263.

4. Gorelick PB, Scuteri A, Black SE, et al. Vascular contributions to cognitive impairment and dementia: a statement for healthcare professionals from the American heart association/American stroke association. Stroke. 2011;42(9):2672-2713.

5. O'Brien JT, Thomas A. Vascular dementia. Lancet. 2015;386(10004):1698-1706.

6. Stern Y. Cognitive reserve in ageing and Alzheimer's disease. Lancet Neurol. 2012;11(11):1006-1012.

7. Hurford R, Charidimou A, Fox Z, et al. Domain-specific trends in cognitive impairment after acute ischaemic stroke. $J$ Neurol. $2013 ; 260(1): 237-241$. 
8. Sobral M, Pestana MH, Paúl C. Cognitive reserve and the severity of Alzheimer's disease. Arq Neuropsiquiatr. 2015;73(6):480-486.

9. Vyas S, Rodrigues AJ, Silva JM, et al. Chronic Stress and Glucocorticoids: From Neuronal Plasticity to Neurodegeneration. Neural Plast. $2016 ; 6391686$.

10. Kalaria RN, Akinyemi R, Ihara M. Stroke injury, cognitive impairment and vascular dementia. Biochim Biophys Acta. 2016;1862(5):915-925.

11. Merriman NA, Sexton E, Donnelly NA, et al. Managing cognitive impairment following stroke: protocol for a systematic review of nonrandomised controlled studies of psychological interventions. BMJ Open. 2018;8(1):e019001.

12. Tang EY, Amiesimaka O, Harrison SL, et al. Longitudinal Effect of Stroke on Cognition: A Systematic Review. J Am Heart Assoc. 2018;7(2).

13. Sun JH, Tan L, Yu JT. Post-stroke cognitive impairment: epidemiology, mechanisms and management. Ann Transl Med. 2014;2(8):80.

14. Mohd Zulkifly MF, Ghazali SE, Che Din N, et al. A Review of Risk Factors for Cognitive Impairment in Stroke Survivors. Scientific World Journal. 2016;2016:3456943.

15. McKay E, Counts SE. Multi-Infarct Dementia: A Historical Perspective. Dement Geriatr Cogn Dis Extra. 2017;7(1):160-171.
16. Iadecola C. The pathobiology of vascular dementia. Neuron. 2013;80(4):844-866.

17. Samir Al-Adawi, Nady Braidy, Musthafa Essa, et al. Cognitive Profiles in Patients with Multi-Infarct Dementia: An Omani Study. Dement Geriatr Cogn Dis Extra. 2014;4(2):271-282.

18. Ganguli M, Fu B, Snitz BE, et al. Vascular risk factors and cognitive decline in a population sample. Alzheimer Dis Assoc Disord. 2014;28(1):9-15.

19. Crum RM, Anthony JC, Bassett SS, et al. Population-based norms for the Mini-Mental State Examination by age and educational level. JAMA. 1993;269(18):2386-2391.

20. Park SH, Sohn MK, Jee S, et al. The Characteristics of Cognitive Impairment and Their Effects on Functional Outcome After Inpatient Rehabilitation in Subacute Stroke Patients. Ann Rehabil Med. 2017;;41(5):734-742.

21. Mok VC, Wong A, Lam WW, et al. Cognitive impairment and functional outcome after stroke associated with small vessel disease. J Neurol Neurosurg Psychiatry. 2004;75(4):560-566.

22. Diamond A. Executive Functions. Annu Rev Psychol. 2013;64:135-168. 